\title{
Characterization of Non-linear Internal Waves Using PIV/PLIF Techniques
}

\author{
M. Mohaghar ${ }^{1 *}$, D. R. Webster ${ }^{1}$ \\ ${ }^{1}$ Georgia Institute of Technology, School of Civil and Environmental Engineering, Atlanta, USA \\ *mohaghar@gatech.edu
}

\begin{abstract}
Internal waves are ubiquitous in the ocean. They often form in regions of high temperature or salinity variability as the pycnocline oscillates to form the wave (Phillips, 1966). They can be generated either from the interaction of tidal currents with submarine bathymetry (Garrett and Kunze, 2007) or by wind stress at the ocean surface (Munk and Wunsch, 1998). The current study addresses non-linear internal waves due to their importance in the mixing and dynamics of both atmospheric and oceanographic flows.

Due to the significance of this phenomena, numerous investigations have been conducted to obtain satisfactory theoretical solutions for internal waves in several types of fluid systems. The verification of these models requires precise and accurate experimental data. It should be noted that such models generally assume simple two-layer stratified system separated by a sharp interface. In reality, there is a gradient of density at the interface of the two layers, which can make both experimental and theoretical analysis more challenging. To date, most experimental studies for several types of internal waves have been performed using ultrasonic probes, conductivity probes, resistance-type wave gauges, or salinity-sensor-type wave gauges, as given by Davis and Acrivos (1967). Koop and Butler (1981). Michallet and Barthelemy (1998) and Umeyama (2002). There is one recent study that used the particle image velocimetry (PIV) technique to determine the Eulerian velocity field of internal waves, but it lacks the detailed density measurements necessary to fully understand the flow (Umeyama and Matsuki, 2011).

The current work aims to fully understand the dynamics of internal waves by measuring the density and velocity fields, and then comparing the experimental results with the theoretical non-linear wave solution. A laboratory-scale apparatus was created to replicate the flow characteristics of internal waves in a twolayer stratified system. An experimental configuration is presented with a density jump of 1.1 and $1.5 \sigma_{t}$ separately. Experiments are conducted in the tank $(2.438 \mathrm{~m} \times 50 \mathrm{~cm} \times 50 \mathrm{~cm})$, which was constructed from clear acrylic sheets with thickness of $1.905 \mathrm{~cm}$. The schematic of the internal wavemaker apparatus is shown in Fig.1(a) (Mohaghar et al., 2020). A line diffuser (PVC) was installed along the middle of the tank floor to be used to fill the tank. A half-cylinder plunger-type wavemaker was used to create a perturbation at the pycnocline represented by the interface between the density layers. On each revolution of the drive mechanism, the switch sent a voltage signal to the external trigger port of a pulse generator. By precisely controlling the delay following the external trigger signal, the pulse generator sent a signal to the Nd:YAG laser and the camera to capture an image at a targeted phase of the wave cycle. Images are recorded with a high resolution 29 MP CCD camera, (14-bits, $6600 \times 4400$ pixels).

PIV was used to measure the velocity field, and the fluids in both layers were seeded with neutrallybuoyant particles. The seeded particles were illuminated using a dual-cavity New Wave Research Gemini PIV laser at wavelength of $532 \mathrm{~nm}$, which is diverged into a sheet. Light entering the PIV camera passed through a $532 \mathrm{~nm}$ bandpass filter. The image pairs were processed with Insight $4 \mathrm{G}^{T M}$ software using a $32 \times$ 32 pixel final spot size with 50\% overlap. A sample of PIV vector field for the case of $\Delta \rho=1.5 \sigma_{t}$ is shown in Fig. 1(b), In order to measure the density fields, the flow is visualized using the planar laser-induced fluorescence (PLIF) method for scalar visualization. A laser-fluorescing dye, Rhodamine 6G, is mixed into the heavier layer and the light sheet is used to fluoresce the dye. Following the procedures outlined by Mohaghar (2019), the dye concentration is then inferred from the digital images. In order to capture only fluorescence emitted by Rhodamine 6G, the camera is equipped with a notch filter blocking the $532 \mathrm{~nm}$ wavelength of light. A sample of a final processed PLIF image for the case of $\Delta \rho=1.5 \sigma_{t}$ is shown in Fig.11(c).
\end{abstract}




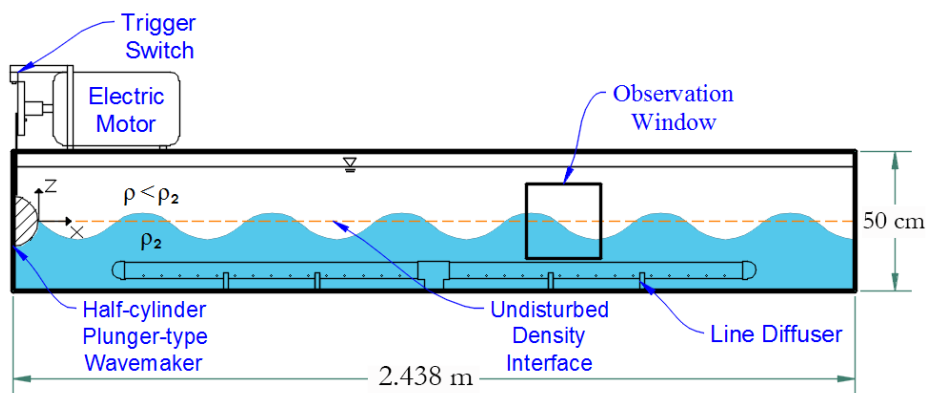

(a)

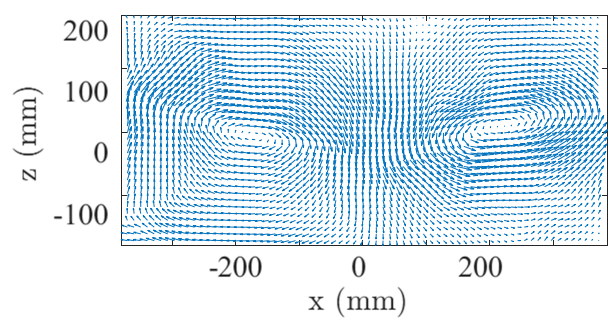

(b)

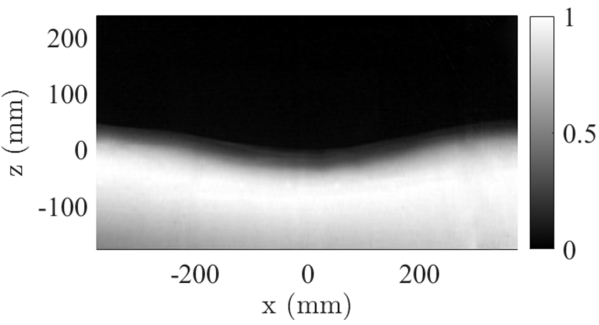

(c)

Figure 1: (a) Schematic diagram of the internal wavemaker apparatus. (b) Sample of PIV vector field. (c) Sample of processed PLIF image. The sample fields shown are at the same phase in the wave cycle.

The interface location, density gradient, wave amplitude and period, velocity and vorticity fields, kinetic energy and shear strain rate are quantified by several phases in one wave cycle and subsequently compared with the corresponding predictions based on third-order Stokes internal-wave theory.

\section{References}

Davis RE and Acrivos A (1967) Solitary internal waves in deep water. Journal of Fluid Mechanics 29:593607

Garrett C and Kunze E (2007) Internal tide generation in the deep ocean. Annu Rev Fluid Mech 39:57-87

Koop CG and Butler G (1981) An investigation of internal solitary waves in a two-fluid system. Journal of Fluid Mechanics 112:225-251

Michallet H and Barthelemy E (1998) Experimental study of interfacial solitary waves. Journal of Fluid Mechanics 366:159-177

Mohaghar M (2019) Effects of initial conditions and Mach number on turbulent mixing transition of shockdriven variable-density flow. Ph.D. thesis. Georgia Institute of Technology

Mohaghar M, Jung S, Haas KA, and Webster DR (2020) Copepod behavior responses around internal waves. Frontiers in Marine Science 7:331

Munk W and Wunsch C (1998) Abyssal recipes II: Energetics of tidal and wind mixing. Deep Sea Research Part I: Oceanographic Research Papers 45:1977-2010

Phillips OM (1966) The Dynamics of the Upper Ocean. Cambridge University Press

Umeyama M (2002) Experimental and theoretical analyses of internal waves of finite amplitude. Journal of Waterway, Port, Coastal, and Ocean Engineering 128:133-141

Umeyama M and Matsuki S (2011) Measurements of velocity and trajectory of water particle for internal waves in two density layers. Geophysical Research Letters 38:L03612 